NOTA TÉCNICA

\title{
VARIABILIDAD GENÉTICA, FENOTÍPICA Y CORRELACIONES ENTRE COMPONENTES DE RENDIMIENTO DE LÍNEAS DE GIRASOL (Helianthus annuus) ${ }^{1}$
}

\author{
Alfredo Ortegón ${ }^{2}$, Artemio Escobedo ${ }^{2}$
}

\begin{abstract}
RESUMEN
Variabilidad genética, fenotípica y correlaciones entre componentes de rendimiento de líneas de girasol (Helianthus annuus). Los objetivos de este trabajo fueron observar la variabilidad genotípica y fenotípica, así como los efectos directos e indirectos en la correlación de seis características sobre el rendimiento de grano de las líneas 285,386,486, CM3 y VI de girasol, las cuales son mantenedoras de la esterilidad citoplásmica. El experimento se estableció en el mes de agosto de 1991 en Río Bravo, Tamaulipas, México bajo condiciones de riego. Los resultados mostraron que en este grupo de líneas existe mayor variabilidad genética en el peso de la semilla, altura de planta y en el período reproductivo. En los caracteres rendimiento de grano y aceite se detectó baja variabilidad genética y baja heredabilidad, pero manifestaron un mejor avance genético esperado. Con el rendimiento de grano correlacionaron alta y positivamente las variables de diámetro de capítulo, peso de 1000 semillas y período vegetativo, y en forma negativa con el rendimiento de aceite. Los análisis de efectos directos e indirectos mostraron que la altura de planta, período vegetativo y el peso de 1000 semillas tuvieron mayor influencia sobre el rendimiento de grano.
\end{abstract}

\begin{abstract}
Genetic and phenotypic variability and correlations between yield components of sunflower (Helianthus annuus) lines. An experiment was conducted in August of 1991 under irrigated conditions at Rio Bravo, Tamaulipas, Mexico, in arder to observe the genetic and phenotypic variance, the direct and indirect correlation effects of six caracters on the grain yield of the 285, 386, 486, CM3 and VI sunflower lines, which are carriers of cytoplasmic mal e sterility. The results, of this group of lines, showed a high genetic variance in seed weight, plant height and reproductive periodo The characters of grain yield and oil content showed low genetic variance and low heritability, but a higher expected genetic adyance. The grain yield was high and positively correlated with the variables of head diameter, weight of 1000 seeds and vegetative period, but negatively to oil yield. The analysis of direct and indirect effects showed that the plant height, vegetative period and weight of 1000 seeds had a higher influence on the grain yield.
\end{abstract}

\section{INTRODUCCIÓN}

Los híbridos de girasol han mostrado buena adaptabilidad en siembras establecidas en el área agrícola del norte y centro del estado de Tamaulipas (México), situación que motivó para que el programa de mejoramiento genético de girasol del Campo Experimental Río BravoINIFAP se avocará en 1980 a la formación de' líneas para la localidad con esterilidad citoplásmica, mantenedoras de la esterilidad y restauradoras de la fertilidad para formar híbridos capaces de competir con los de importación.

Shabana (1974) comenta la necesidad de realizar investigación en particular de girasol en un rango amplio de ambientes, acerca de la variabilidad genética de cada parámetro y su asociación entre ellos mismos para crear un

1 Presentado en la XL Reunión Anual del PCCMCA en Costa Rica, América Central. 13 al 19 de marzo, 1994.

2 Investigadores del Programa de Oleaginosas. Campo Experimental Río Bravo-INIFAP. Apartado Postal NºI72, Río Bravo, Tam., México. CP. 88900. 
modelo para altos rendimientos. Tyagi (1988) indica que es imperativo determinar el valor relativo de diversos efectos genéticos en el material progenitor antes le iniciar un extensivo programa de mejoramiento para desarrollar híbridos adaptados para áreas narginales.

Estudios sobre variabilidad genética de componentes del rendimiento en girasol, así como correlaciones y sus análisis sobre efectos directos e indirectos entre dichas variables han sido reali:ados por diversos investigadores (Green 1980; vanov y Stoyanova 1980; Marinkovic y Skhoric 988; Marjanac 1988; Tyagi 1988).

Los objetivos de este trabajo fueron observar la variabilidad genotípica y fenotípica, así como los efectos directos e indirectos en la correación de seis características sobre el rendimiento de grano de cinco líneas (B) de girasol mantenedoras de la esterilidad citoplásmica.

\section{MATERIALES Y MÉTODOS}

El trabajo se realizó en el Campo Experimental Río Bravo (CERIB) del Instituto Nacional le Investigaciones Forestales y Agropecuarias INIF AP) de México, localizado en el norte del stado de Tamaulipas, en los $26^{\circ}$ latitud norte y $18^{\circ}$ longitud oeste, y con altura de $32 \mathrm{msnm}$.

El material experimental utilizado fueron inco líneas (B) mantenedoras de la esterilidad itoplásmica: 285,386,486, CM3 y V1, obtenidas n el CERIB. La siembra se realizó en agosto de 991 en un suelo areno-arcilloso en surcos separados a $80 \mathrm{~cm}$ y con una distancia entre plantas e $25 \mathrm{~cm}$; como parcela experimental se pusie-:m cuatro surcos de $5 \mathrm{~m}$ de largo y su distribución se efectuó en un diseño de bloques al azar on cuatro repeticiones.

Se fertilizó con la fórmula comercial, 80-40-0. Se aplicaron dos riegos de auxilio a los 30 y 5 días de nacidas las plantas y dos deshierbas.

Se tomaron datos de altura de planta (AP) y iámetro de capítulo (DC) en centímetros; período vegetativo (PV) y período reproductivo (PR) en días; peso de 1000 semillas (P1000S) en gramos y rendimiento de grano $(R G)$ y rendimiento de aceite (RA) en kilogramos por hectárea.

El análisis estadístico se realizó para cada una de las variables consideradas. Los componentes de varianza genotípica y fenotípica para cada carácter se calcularon de los cuadrados medios del análisis de varianza descrito por Miller et al. (1958). La heredabilidad en sentido amplio se estimó al dividir la varianza genética entre la varianza fenotípica y multiplicada por 100. Los coeficientes de varianza genética y fenotípica se calcularon al dividir la raíz cuadrada de los mismos por la media de la población y multiplicada por 100. La predicción del avance genético esperado se calculó por la fórmula $\mathrm{AGE}=\mathrm{S}^{2} \mathrm{~g} / \mathrm{Sph} \mathrm{x} \mathrm{k}$; donde $\mathrm{S}^{2} \mathrm{~g}$ es la varianza genética, Sph es la desviación estándar fenotípica y $\mathrm{k}$ es el diferencial de selección que para una intensidad de selección del $5 \%$ para una característica enparticular tuvo una constante de 1,16.

Las correlaciones genotípica y fenotípica entre el rendimiento de grano con las otras características registradas se determinaron por la varianza y covarianza. Se practicó a su vez el análisis de efectos directos e indirectos.

\section{RESULTADOS Y DISCUSIÓN}

Los valores presentados en el Cuadro 1 muestran las variaciones obtenidas en las siete características estudiadas en su rango, media, desviación estandar (Sx) y varianza genética $\left(S^{2} \mathrm{~g}\right)$ y fenotípica $\left(S^{2} \mathrm{ph}\right)$. Es evidente de acuerdo a estos resultados que ocurrió mayor variación para P1000S y para AP seguida por PR, esta variación se aprecia de mejor manera expresada por los coeficientes de varianza gen ética (CVG) y varianza fenotípica (CVP) señalados en el Cuadro 2 el P1000S obtuvo el mayor CVG $(19,1)$ seguido por AP $(18,4)$ y el PR $(11,6)$. Por otra parte la diferencia mayor entre los valores obtenidos para CVG y CVP se detectó para RG y RA.

La heredabilidad calculada en sentido amplio de la relación entre la $\mathrm{S}^{2} \mathrm{~g}$ y $\mathrm{S}^{2}$ ph detectó un alto valor de 86,8 
Cuadro 1. Rango, media, desviación estándar y componentes de varianza genética y fenotípica de siete características cuantitativas en líneas B de girasol. Río Bravo,Tam. 1991.

\begin{tabular}{lcrccc}
\hline Característica & Rango & Media & Sx & $\mathbf{S}^{2} \mathbf{g}$ & $\mathbf{S}^{2} \mathbf{p h}$ \\
\hline RG (kg/ha) & $1147-1507$ & 1275 & 108 & 9813 & 56083 \\
RA (kg/ha) & $498-537$ & 514 & 44 & 1672 & 5914 \\
P1000S (g) & $44-75$ & 58 & 3,8 & 123 & 183 \\
DC (cm) & $13-15$ & 14 & 0,48 & 0,73 & 1,58 \\
AP (cm) & $80-133$ & 109 & 3,91 & 400 & 461 \\
PV (días) & $63-72$ & 67 & 1,22 & 12 & 18 \\
PR (días) & $25-33$ & 29 & 1,16 & 11,4 & 16,8 \\
\hline
\end{tabular}

Cuadro 2. Heredabilidad (H2\%), coeficiente de varianza genética (CVG) y fenotípica (CVP), avance genético esperado (AGE) y avance genético esperado como porcentaje de la media de siete características de líneas B de girasol. Río Bravo, Tam. 1991.

\begin{tabular}{lccccc}
\hline Característica & $\begin{array}{c}\text { H2 } \\
(\boldsymbol{\%})\end{array}$ & $\begin{array}{c}\text { CVG } \\
(\boldsymbol{\%})\end{array}$ & $\begin{array}{r}\text { CVP } \\
(\boldsymbol{\%})\end{array}$ & $\begin{array}{c}\text { AGE* } \\
\mathbf{k = 1 , 1 6}\end{array}$ & $\begin{array}{c}\text { AGE } \\
(\boldsymbol{\%})\end{array}$ \\
\hline RG & 17,5 & 7,8 & 18,6 & 48,1 & 3,8 \\
RA & 28,3 & 8,0 & 15,0 & 25,2 & 4,9 \\
P1000S & 67,2 & 19,1 & 23,3 & 15,7 & 27,6 \\
DC & 46,2 & 6,1 & 9,0 & 0,7 & 0,05 \\
AP & 86,8 & 18,4 & 19,7 & 21,6 & 19,8 \\
PV & 66,7 & 5,2 & 6,3 & 3,3 & 0,5 \\
PR & 67,9 & 11,6 & 14,1 & 3,2 & 4,1 \\
\hline
\end{tabular}

* Valor de $\mathrm{k}(1,16)$ con base en selección al $(5 \%)$ de una característica determinada.

$\%$ para AP, y un valor medio para PR, PV y P1000S con $67,9 \%, 67,2 \%$ y $66,7 \%$ en forma respectiva. Estas cuatro características fueron menos variables bajo las condiciones de esta prueba, por lo que se puede considerar que la selección fenotípica en este grupo de líneas podría ser efectiva para estas características. La altura de planta y el peso de 1000 semillas al detectar un alto avance genético esperado indicó que la heredabilidad en estas dos cara terísticas fue por acción génica aditiva, mientras que para PV y PR con menor avance genético esperado, la heredabilidad se debió a efectos no aditivos.

El diámetro de capítulo por su menor variabilidad, baja heredabilidad y mínimo avance genético esperado, indicó que para este carácter la selección sería poco efectiva en estas líneas.

Los rendimientos de grano y de aceite al mostrar baja heredabilidad y bajo CVG pudo ser resultado de una uniformidad en el potencial genético de este grupo de líneas y poco se podría lograr por selección para mejorar ambas características, no obstante por su valor en el AGE estas líneas podrían mostrar mayor efectividad en el RG y RA en combinaciones híbridas. Estos resultados en relación a las características de AP, RG y RA coinciden con lo obtenido por Ivanov y Stoyanova (1980); y con Baez et al. (1988) quienes obtuvieron baja heredabilidad para rendimiento de grano y rendimiento de aceite y alta 
heredabilidad para altura de planta. A la vez contrastan con resultados obtenidos por Shabana (1974) que encontró alta heredabilidad en las variables rendimiento de grano y rendimiento de aceite.

Resultados de este trabajo deben considerarse con cierta reserva ya que son datos de un solo año y una sola localidad, lo que implica posibles desviaciones en los valores de componentes de varianza.

En el Cuadro 3 se señalan los valores de correlación genotípica (rg) y correlación fenotípica (rp) en las características consideradas en este trabajo. Es notable el alto valor de correlación genotípica positiva $(0,929)$ obtenida entre RG y DC y la correlación negativa $(-0,740)$ con RA. Por el contrario el valor fenotípico en la relación $\mathrm{RG}$ con RA fue alta y positiva $(0,848)$, mientras que con el DC fue no significativa.

El rendimiento de grano correlacionó alta y positivamente con P1000S y con PV genotípicamente (0,755 y $0,754)$, mientras que valores medios de correlación se de- tectaron fenotípicamente $(0,434$ y 0,531$)$. La altura de planta mostró baja correlación positiva en ambos casos.

El rendimiento de aceite mostró genotípicamente una correlación media negativa con DC $(-0,654)$.y positiva fenotípicamente $(0,536)$.

La altura de planta en ambos casos mostró una correlación media positiva con DC $(0,673$ y 0,500$)$.

El período reproductivo mostró valores negativos en ambos casos con todas las características incluidas menos con RA, donde se detectaron las relaciones genotípicas PRIPV, PR/DC y PRIAP con valores -0,917, -0,862 y $-0,662$ en forma respectiva y la relación fenotípica PR/PV con valor de -0.920 .

Dewey y Lu (1959) hacen especial recomendación en utilizar la correlación genotípica para evitar repeticiones innecesarias. Martinov citado por Ivanov y Stoyanova (1980) con relación a lo mismo insisten en que la correlación genotípica es la base para el análisis de efectos

Cuadro 3. Matriz de correlaciones genotípicas (rg) y fenotípicas (rp) de rendimiento de grano y componentes de rendimiento de líneas B de girasol. Río Bravo, Tam. 1991.

\begin{tabular}{lllllll}
\hline Característica & RA & P1000S & DC & AP & PV & PR \\
\hline RG rg & $-0,740$ & 0,755 & $0,929^{* *}$ & 0,281 & 0,754 & $-0,271$ \\
rp & $0,848^{*}$ & 0,434 & 0,040 & 0,257 & 0,531 & $-0,480$ \\
RA rg & $-0,055$ & $-0,654$ & $-0,073$ & 0,033 & 0,123 & \\
rp & 0,481 & 0,536 & 0,136 & 0,285 & $-0,076$ & \\
P1000S rg & & $-0,184$ & $-0,125$ & $-0,391$ & $-0,144$ & \\
$\quad$ rp & 0,015 & $-0,127$ & 0,281 & $-0,076$ & \\
DC rg & 0,673 & 0,611 & $-0,862^{*}$ & & \\
rp & 0,500 & 0,312 & $-0,333$ & & \\
AP rg & 0,408 & $-0,662$ & & & \\
rp & 0,366 & $-0,534$ & & & \\
PV rg & & $-0,917 * *$ & & & \\
$\quad$ rp & & $-0,920^{* *}$ & & & \\
\hline
\end{tabular}


directos e indirectos donde la separación de las características dan mayor claridad que en el nivel fenotípico.

Bajo estas consideraciones los análisis de efectos directos e indirectos de estas características en relación a rendimiento de grano se realizaron en su nivel genotípico. En el Cuadro 4 se detallan los valores calculados correspondientes a los efectos directos e indirectos.

P1000S, AP, PV y PR mostraron un efecto directo positivo con el rendimiento de grano, mientras que RA y DC marcaron un efecto directo negativo.

El efecto directo en su relación RG/RA mostró alto valor negativo $(-4,1754)$, que fue atenuado principalmente por los efectos indirectos de DC y AP, con un apoyo leve de los efectos indirectos PV y PR; aún así la correlación fue alta y negativa $(-0.740)$.

La relación RG/P1000S mostró efecto directo positivo $(1,0441)$, que tuvo como principal efecto indirecto positivo en el DC, seguido por el RA. Como principales efectos indirectos negativos resultaron el PV seguido por AP.

El diámetro de capítulo cuya correlación con RG fue alta y positiva $(0,929)$, manifestó alto efecto directo negativa $(-4,7328)$, reforzado por los efectos indirectos negativos del PR $(-0,5312)$ y P1000S $(-0,1922)$. El balance po- sitivo de la correlación estuvo determinada por los efectos indirectos positivos de RA, AP y PV con valores de $2.7307,2.2847$ y 1.3696 en forma respectiva.

La altura de planta mostró alto efecto directo positivo (3.3948) con apoyo del efecto indirecto positivo de PV. Las otras características expresaron efectos indirectos negativos donde sobresalió DC (-3.1852).

El período vegetativo mantuvo alto efecto directo positivo con apoyo en los efectos indirectos positivos de AP y PR. Las otras características con su efecto indirecto negativo donde predominó el DC balancearon esta correlación.

El período reproductivo mostró bajo efecto directo positivo, el cual fue superado ampliamente por el efecto indirecto positivo de DC (4.0797). Las otras cuatro características marcaron efectos indirectos negativos donde predominaron en su balance AP y PV.

Para corregir los efectos directos negativos del rendimiento de aceite y diámetro de capítulo, es factible que la selección para aumentar el peso de semilla y el período reproductivo puedan ser considerados.

Estos resultados en su efecto directo genotípico coinciden con lo obtenido por Ivanov y Stoyanova (1980) en las características RA, P1000S, DC y AP, y difieren en el PV y PR.

Cuadro 4. Efectos directos e indirectos de seis características y su influencia en el rendimiento de grano de líneas B de girasol. Río Bravo, Tam. 1991.

\begin{tabular}{lcccccc}
\hline Característica & RA & P1000S & DC & AP & PV & PR \\
\hline RA & $-4,1754$ & 0,2297 & 2,7307 & $-0,3048$ & $-0,1378$ & $-0,5136$ \\
P1000S & $-0,0574$ & 1,0441 & $-0,1922$ & $-0,1305$ & $-0,4082$ & $-0,1504$ \\
DC & 3,0952 & 0,8708 & $-4,7328$ & $-3,1852$ & $-2,8917$ & 4,0797 \\
AP & 0,2478 & $-0,4244$ & 2,2847 & 3,3948 & 1,3851 & $-2,2474$ \\
PV & 0,0740 & $-0,8765$ & 1,3696 & 0,9146 & 2,2416 & $-2,0556$ \\
PR & 0,0758 & $-0,0887$ & $-0,5312$ & $-0,4079$ & 0,5651 & 0,6162 \\
\hline rg/RG & $-0,740$ & 0,755 & 0,929 & 0,281 & 0,754 & $-0,271$ \\
\hline
\end{tabular}




\section{CONCLUSIONES}

1. En este grupo de líneas existe mayor variabilidad genética en el peso de semilla, altura de planta y período reproductivo.

2. En los caracteres rendimiento de grano y rendimiento de aceite se detectó baja variabilidad genética y baja heredabilidad, pero manifestaron mejor avance gen ético esperado.

3. Con el rendimiento de grano correlacionaron alta y positivamente los parámetros de diámetro de capítulo, peso de 1000 semillas y período vegetativo, y en forma negativa con el rendimiento de aceite.

4. Los análisis de efectos directos e indirectos mostraron que los caracteres de altura de planta, período vegetativo y el peso de 1000 semillas tuvieron mayor influencia sobre el rendimiento de grano.

\section{LITERATURA CITADA}

BAEZ, M. 1.; LUDUEÑA, P.; SANGUINETII, A 1988. Correlaciones fenotípicas, genotípicas y ambientales entre caracteres de girasol y podredumbre del tallo (Macrophomina phaseoli (Maub.) Ashby). 12th Int. SunfIower Conf. Novi Sad, Yugoslavia. 11. pp. 519-524.

DEWEY, D.; LV, K. 1959. A correlation and path-coefficient analysis of components of crested wheatgrass seed production. Agron. J.51: 515-518.
GREEN, V. E. 1980. Correlation and path coefficient analysis of the components of yield in sunflower cultivars Helianthus annuus L. IX Conf. Int. del girasol. Malaga, España. 11. pp. 12-21.

IVANOV, P.; STOYANOVA, Y. 1980. Studies on the genotypic and phenotypic variability and some correlations in sunflower (Helianthus annuus L.). IX Conf. Int. del girasol. Malaga, España. 1. pp. 336-342.

MARINKOVIC, R.; SKHORIC, D. 1988. Path coefficient analysis of components of sunflower seed yield $(H$. annuus L.) 12th Int. Sunflower Conf. Novi Sad, Yugoslavia. 1. pp. 406-407.

MARJANAC, D. 1988. Direct and indirect effects of variables on grain yield of some novi sad sunflower hibrids.12th Int. Sunflower Conf. Novi Sad, Yugoslavia. 1. pp 410412.

MILLER, P. A; WILLIAMS, J. C.; ROBINSON, H. F.; COMSTOCK, R. E. 1958. Estimates of genotypic and enviromental variances and covariances in upland cotton and their implications in selection. Agron. J. 50: 126-131.

SHABANA, R. 1974. Genetic variability of sunflower varieties and inbred lines. 6th Int. Sunflower Conf. Bucharest, Rumania. pp 263-269.

TYAGI, A P. 1988. Combining ability analysis for yield components and maturity traits in sunflower (Helianthus annuus L.). 12th Int. Sunflower Conf. Novi Sad, Yugoslavia. 11. p.489-493. 